\title{
Optimal Energy Consumption for Wireless Sensor
}

\section{Networks}

\author{
Jang, Hung-Chin \\ Lee, Hon-Chung \\ Huang, Jun-Xiang \\ Department of Computer Science, National ChengChi University, Taiwan, R.O.C.
}

\begin{abstract}
Due to congenital restriction of sensors, it is always a crucial issue on how to utilize limited energy effectively. We first put forward a network topology construction method, based on energy saving, including the way of forming a cluster and selecting middle heads and cluster heads. We then employed Simulated Annealing method to optimize energy consumption of the whole system. Results show that the proposed method is able to evenly distribute energy consumption among sensors, lengthen the lifetime of each sensor, prolong network lifetime, and increase network transmitted data volume.
\end{abstract}

Keywords: wireless sensor network, energy consumption, simulated annealing, optimization

\section{Introduction}

Sensors have the capabilities of doing sensing, data processing, and wirelessly transmitting collected data back to base stations by way of multiple-hop relay. Sensor itself supplies necessary operation with limited battery energy. Those operations that consume energy are transmitting and receiving data, running applications, measuring power, and even staying in standby mode. Among others, data transmission consumes the most energy. In a sensor network, network lifetime is important for applications. Energy consumption of each sensor directly affects the network operational lifetime. Those sensors which close to base stations consume more energy since they should relay data for more sensors, and thus are apt to reduce their lifespan while being bad. Neighboring sensors of those bad sensors must relay data with stronger power, the energy consumption of these sensors is even quicker.

In this research, we put forward an effective method to manage cluster topology and use Simulated Annealing to optimize the energy saving. The objectives of this research are to average the energy consumption of sensor nodes, lengthen the lifetime of each sensor node, prolong network lifetime, and optimize data volume transmission of the whole system.

\section{Systematic Architecture}

Sensor network consists of base stations and sensors. Base stations serve as gateways to exchange data and control information with other networks. All the distributed sensors monitor and gather environmental data, and pass back the collected data to base stations for further processing. Clustering is a very important concept on sensor networks. We classify neighboring sensors into the same cluster such that the data collected from these sensors can be fused [1] first and then sent out. A sensor network consists of a couple of clusters. Each cluster has a cluster head which collects and fuses data (composite bit-stream) from its cluster members. The fused data will then be sent via multi-hop hierarchy to other cluster heads, and then to base stations [2].

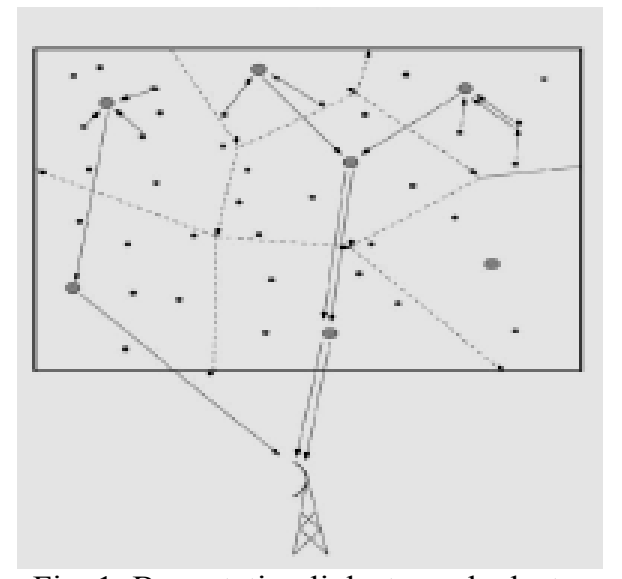

Fig. 1: Base station links to each cluster hierarchically from near to far.

\subsection{Set-up phase}

Set-up phase is used to construct or rebuild network. Base station will link to each cluster hierarchically, from near to far, and complete the whole topology at last (Fig. 1). This phase can be divided into seven steps:

[Step1] Each node is determined to be either a cluster head or a cluster member according to a certain probability.

[Step2] BS executes Inquiry to find out whether there are any newly discovered 1-hop-distance cluster heads.

[Step3] BS executes Page to link to all those 
1-hop-distance heads. All these heads record the address of base station as PFA (Packet Forward Address).

Initial value $\mathrm{k}=1$ (hop count)

[Step4] All k-hop-distance heads turn off scan mode to prevent mutually inquiry.

[Step5] The head in k-hop-distance begins Inquiry to look for the following kinds of sensors:

- All those cluster heads have not been found out by (k-1)-cluster heads

- Members in the k-hop-distance

If none of cluster heads has been found, go to [Step7].

[Step6] Each k-hop-distance head links to each (k $+1)$-hop-distance head. Each $(\mathrm{k}+1)$ - hop-distance head records at least one PFA of k-hop-distance, and then turn off scan mode.

[Step7] Each k-hop-distance cluster head together with all members linked to this head form a cluster.

If it skips [Step6] then finishes set-up phase, otherwise, executes $\mathrm{k}++$ and go to [Step5].

\subsection{Steady-state phase}

After set-up phase, the system enters steady-state phase. In this phase, each cluster member sends monitored data to its cluster head. Cluster head will fuse or compress the collected data first, and then send to the cluster head that close to the base station. After a while, the system may enter set-up phase again to reconstruct the network architecture if the topology changed due to sensors movement or failure.

\section{Methodology}

If the system uses either random or take-turns method to make head selection, or if the head is not located at the center of the cluster, but far away from the troop, all the sensors (both heads and members) in such cluster should spend more power in transmitting data. Here we choose the most effective cluster head to reduce power consumption and thus lengthen its lifetime. Besides, we add middle heads in the cluster to link those members which are far away from head (Fig. 2) to have more energy saving.

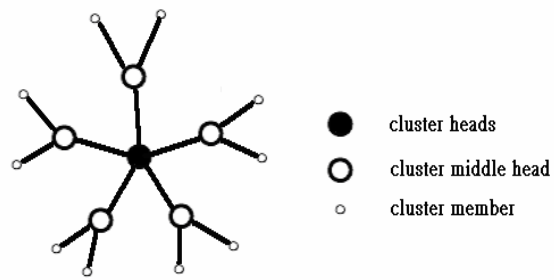

Fig. 2: Middle head is used to link other members.
As to choose an effective cluster head and its corresponding middle heads is a NP-Problem. Here we propose an approach to select cluster heads by using Simulated Annealing (SA). Calculate the mean of residual energy of nodes in cluster and check to see if it satisfies the residual energy constraint. Add "middle heads" to reduce the energy consumption in long-distance transmissions. The residual energy constraint is as follows:

$E_{\text {mean }}-\sigma \leqq E_{\text {resid }} \leqq E_{\text {mean }}+\sigma$

where $E_{\text {resid }}$ is the residual energy of cluster head,

$E_{\text {mean }}$ is the mean of residual energy of all nodes in cluster, and $\sigma$ is a constraint constant.

The cluster is established randomly at the first round as the network comes to set-up phase. Next time the system comes to reconstruct the network. The derived cluster in the previous round will be transformed into a tree, which is the initial solution of the next round computation.

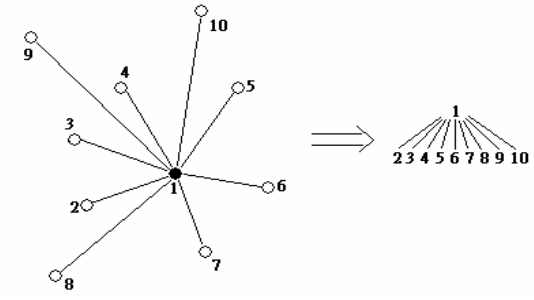

Fig. 3: Transform a cluster into a tree.

We then add an empty subtree to each node of the tree, which results in the initial solution of SA. In Fig. 4 we take node 2 or node 3 as the initial solution of cluster head.
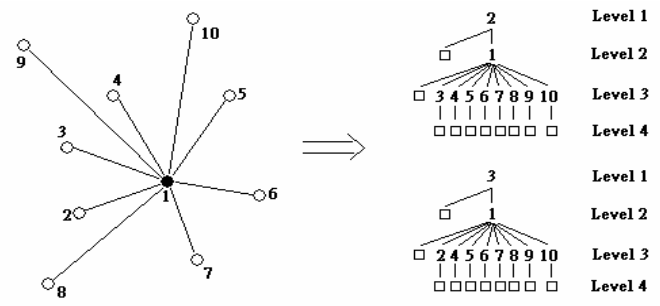

Fig. 4 : Derive an initial solution.

In Fig. 5, step1 is the initial solution. Step2 exchanges the subtree (node 9) of node1 with the subtree (an empty tree) of node3. Step3 exchanges subtree (node 10) of nodel with subtree (an empty tree) of node4. Step4 exchanges subtree (node 9) of node 3 with subtree (an empty tree) of node 4 . Step 4 is the derived solution in this round, and could be transformed back to network topology as shown in Fig. 6. 


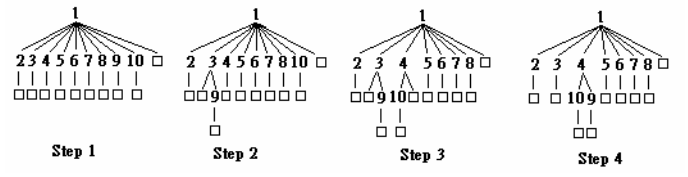

Fig. 5: Derive new solutions

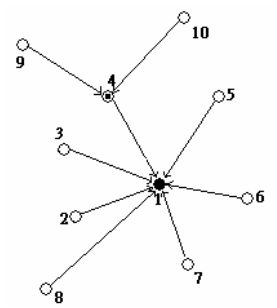

Fig. 6: Network topology of the tree solution.

\subsection{Formulas and Parameters}

The energy consumption formula of transmitting and receiving data between two sensors is as follows [3]:

Energy consumption of transmitting data:

$E_{T x}(k, d)=$ Eelec $^{*} k+$ Camp $^{*} k * d^{2}, d>1$

Therefore, the power consumption of data transmission between two sensors is proportional to the square of their distance. Energy consumption of receiving data:

$E_{R x}(k)=$ Eelec $^{*} k$

while $k$ is the data volume to be transmitted (bit), $d$ is the distance between two sensors, Eelec is the energy consumption to carry out data transmission in terms of $\mathrm{nJ} / \mathrm{bit}$, Camp is the energy consumption constant used to expand radio coverage in terms of $\mathrm{nJ} /\left(\mathrm{bit}^{*} \mathrm{~m}^{2}\right)$.

Total consumed energy of each cluster

$=\Sigma E_{R x}+\Sigma E_{T x}$

$=$ Total consumed energy of data receiving + total consumed energy of data transmitting

The object function (Minimization) of this energy saving problem is:

Objective Function (Cluster Tree)

$=\{$ Eelec $* k *[N(m)+2 * N(m e)] *(R / I)\}+$

$\sum_{i=1}^{N(m)}\left[\left(C_{i}+1\right) *\left(\right.\right.$ Eelec $^{*} k+$ Camp $\left.\left.^{*} k * d_{l i}{ }^{2}\right) *(R / I)\right]$

$+\sum_{j=1}^{N(m e))}\left[\left(\right.\right.$ Eelec $^{*} k+$ Camp $\left.\left.^{*} k * d_{2 j}^{2}\right) *(R / I)\right]$

This objective function consists of three parts, the first part is the total energy consumed of receiving data, the second part is the total energy consumed of transmitting data from cluster middle heads to cluster head, and the last part is the total energy consumed of transmitting data from cluster members to cluster middle heads. Among them, $N(m)$ is the number of middle heads in the cluster. $N(m e)$ is the number of cluster members in the cluster. $C_{i}$ is the number of cluster members linked to middle heads of cluster $i . d_{l i}$ is the distance between middle head $i$ and its cluster head. $d_{2 j}$ is the distance between cluster member $j$ and its middle head. $R$ is the time interval of re-clustering. $I$ is the time interval of transmission.

\subsection{Simulated Annealing}

Simulated Annealing is a combinatorial optimization algorithm attempting to force a system to its lowest energy state through controlled cooling.

The algorithm of using SA to optimize energy saving is as follows:

(1) BestEnergy = Objective Function(Cluster Tree)

(2) For $(i=0 ; i<n ; i++)\{$

(3) if $\left(E_{\text {mean }}-\sigma \leqq E i \leqq E_{\text {mean }}+\sigma\right)$

(4) For (Temperature $=T_{0}$; Temperature $>$ Stop ;

Temperature * grad $)\{$

(5) For $(x=0 ; x<$ Stay_Time ; $x++)\{$

(6) Randomly choose two subtrees to exchange

for a new tree

(7) Energy_temp $=$ Objective Function (new tree)

(8) if (Energy_Temp $\leqq$ BestEnergy) \{

(9) Upgrade new tree

(10) BestEnergy $=$ Energy_temp

(11) if (EnergyTemp $\geqq$ BestEnergy) \{

(12) There is probability e(- $\triangle \mathrm{Z} /$ Temperature)

to update new tree and

(13) BestEnergy $=$ Energy_temp $\}$

(14) $\}$

(15) $\}$

(16)\}

BestEnergy in Step (1) is the initial target value used to compare which topology consumes less energy. Step (2) derives the optimal solution by selecting different heads. In the optimal solution, the root of the new tree is the head, leaves are members, and those nodes neither head nor members are middle heads. Step (3) checks whether the residual energy of node $i$ satisfies the given constraint, where $E i$ is the residual energy of node $i, E_{\text {mean }}$ is the average energy of those nodes in the same cluster, $\sigma$ is a constraint relevant to sensor networks). $T_{0}$ in Step (4) is the initial temperature of SA, Stop is the stop constraint, grad is the cooling speed. Stay Time in Step (5) is the time spent at each temperature (Temperature). In Step (6), we randomly chose two subtees in the new tree for exchange, and then calculate new target value and store in Energy temp in Step (7). For those two nodes (a pair) that either 
belongs to the same parent or both are empty, are not allowed to exchange. Step 8, 9 and 10 check whether new target value is less than old target value, if yes, then update the optimal solution by the current new tree, otherwise, Step 11 and 12 will have the probability of $\mathrm{e}^{(-\Delta \mathrm{Z} / \text { Temperature })}$ to update the solution, which helps to jump out of local trap.

\section{Simulation Results and Analysis}

In the simulation, we set that there are 200 sensors randomly distributed in an $100 \mathrm{~m} \times 100 \mathrm{~m}$ sensor network with any two nodes transmission distance less than $15 \mathrm{~m}$. Each sensor has the probability of 0.2 to be a cluster head, so the number of cluster heads is about 40, i.e. sensor network can be divided into 40 clusters, or equally, there are 5 sensors in each cluster on average. The transmitted message length $(\mathrm{k})$ is 1000 bit, the consumed energy (Eelec) is $50 \mathrm{~nJ} / \mathrm{bit}$, the energy consumed in expanding radio coverage (Camp) is 100 $\mathrm{nJ} /\left(\mathrm{bit}^{*} \mathrm{~m}^{2}\right)$, and each sensor is powered by $0.5 \mathrm{~J}$.

We compare our method with Direct Communication (DC) and Low Energy Adaptive Clustering Hierarchy (LEACH) [3] in terms of network lifetime and collected data volumes. Fig. 7 shows the performances of network lifecycles applying DC, LEACH and SA three different mechanisms, respectively. $\mathrm{X}$ axle coordinate is a time axle in terms of number of computation rounds, and $\mathrm{Y}$ axle coordinate is the number of survival sensors. DC is of single hop architecture and its cluster heads are chosen randomly. Figure shows that half of the sensors die less than 20 rounds, the active range is reduced greatly and ends up with poor performance. LEACH is of multi hop architecture, its members serve as cluster heads in turns. In LEACH, cluster heads do not need to make distant transmissions, its performance efficiency is much better than that of DC. Experiment shows that sensors died in succession rapidly during 500 to 600 rounds. Figure 7 shows the curve of SA is in the form of ladder rather than a smoothed curve. This means that, in the same cluster, the energy consumption of each sensor is almost the same, and the death time of each sensor is very close, which is most significant between 700 and 800 rounds. Using SA the lifecycle of sensors is $25 \%$ longer than that of LEACH. Fig. 8 shows how DC, LEACH, and SA influence on collected data volume. $\mathrm{X}$ axle coordinate is a time axle (round) and $\mathrm{Y}$ axle coordinate represents collected data volume. It shows that SA can significantly increase the collected data volume by at least $20 \%$.

\section{Conclusion}

In this paper, we proposed a topology control method based on clustering, and used Simulated Annealing to optimize energy saving in wireless sensor networks. Experiments show that the proposed method is able to average the energy consumption of sensor nodes, lengthen the lifetime of each sensor node, prolong network lifetime, and optimize data volume transmission of the whole system.

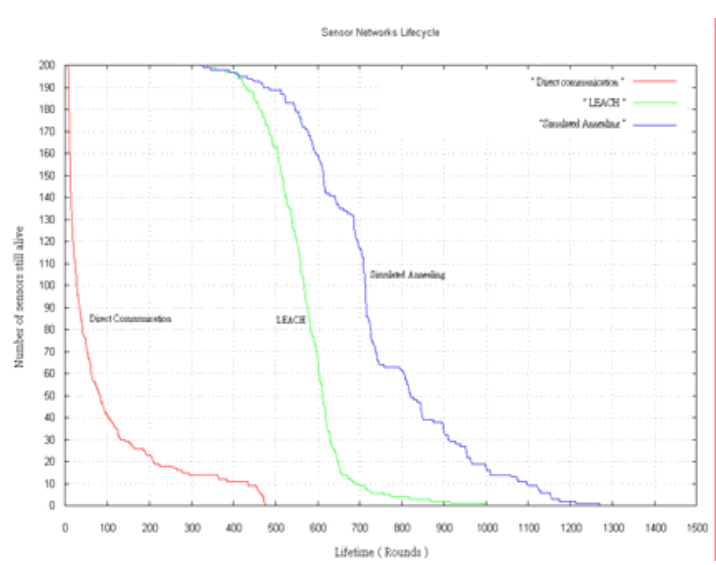

Fig. 7: Comparisons of network lifecycles using three different mechanisms.

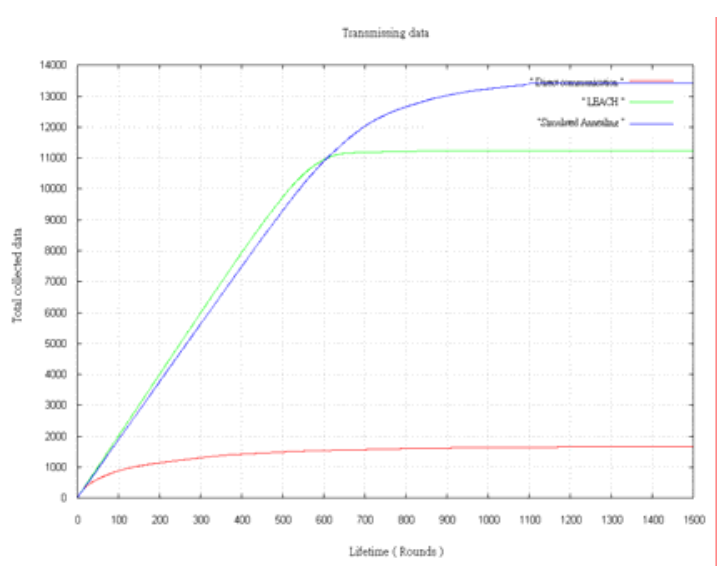

Fig. 8: Comparisons of collected data volumes using three different mechanisms.

\section{References}

[1] L. Klein, "Sensor and data fusion concepts and applications," SPIE Optical Engr Press, WA, 1993.

[2] Matthias Handy, Frank Grassert, Dirk Timmermann, "DCP: A new data collection protocol for bluetooth-based sensor networks," 2004.

[3] W. Heinzelman, A. Chandrakasan and H. Balakrishnan, "Energy-efficient communication protocol for wireless microsensor networks," Proc. of the 33rd International Conference on System Sciences (HICSS '00), 2000. 